\title{
WORLD HEALTH ORGANIZATION EXPERT COMMITTEE ON RHEUMATIC DISEASES, FIRST REPORT, 1954
}

\section{Members of the Committee}

Dr. W. S. C. Copeman (London), Chairman. Prof. F. Coste (Paris).

Prof. G. Edström (Lund).

Prof. J. Goslings (Leyden), Rapporteur.

Prof. A. Ruiz Moreno (B. Aires), Vice-Chairman.

DR. E. T. CONYBEARE (London).

DR. A. HöJER (Trivandrum).

Dr. J. S. Peterson (W.H.O.).

DR. T. S. SzE (W.H.O.), Secretary.

\section{W.H.O. Expert Committee on Rheumatic Diseases.}

First Report.* April, 1954. Wld Hlth Org. techn.

Rep. Ser. No. 78. Published in English and

French. Pp. 25. (25 cts; Swiss frs $1 ; 1$ s. 9d.)

Chronic rheumatic diseases have been somewhat neglected from both a medical and a research viewpoint, despite their social and economic significance as causes of long-term disability. At present there is lack of knowledge concerning the aetiology of this group of diseases, and treatment is therefore still largely palliative and prevention impossible.

A review from a public-health standpoint of the chronic rheumatic diseases of articular and nonarticular types is presented in a recent Technical Report prepared by a W.H.O. expert committee on this subject. It is noted in the report that, as an international health problem, the rheumatic diseases must of necessity be considered less important in some countries than illnesses of an infective or parasitic nature which are amenable to specific measures of control.

\section{Nomenclature and Classification}

Both a nomenclature and a classification are needed for the group of diseases termed "rheumatic". The task of formulating a nomenclature has been undertaken by a special committee of the International League against Rheumatism. As for a classification, any which might be made would have to be considered provisional, since there are as yet too many unknowns in the aetiology and pathogenesis of rheumatic diseases.

\footnotetext{
* This review is reprinted from Chron. Wld Hlth Org., 8, 142.
}

The following general statement is made in the report:

"Rheumatic diseases affect the locomotor system, in which they are important causes of pain, dysfunction, and anatomical change. The most important link between them is now considered to be that they are all diseases peculiar to the connective tissue and that, as such, they all show reactions peculiar to this tissue and especially of its collagen element. It should be emphasized that, although the etiology of the different rheumatic diseases is probably extremely diverse, these connective tissue reactions are common to all of them; in this connexion it should be noted that not only connective tissue of the locomotor system, but also to some extent that of the viscera, the nervous system, the haematopoietic system, the skin, etc., may be affected by these diseases."

In an appendix to the report is a list of the diseases commonly accepted as rheumatic and of other diseases presenting rheumatic features.

\section{Incidence and Prevalence}

Existing data on the incidence and prevalence of chronic rheumatic diseases are not adequate for any of the purposes for which morbidity statistics are commonly used. Four methods have been used to obtain such data as do exist: notification, analysis of medical sickness records (such as health insurance forms), analysis of hospital records, and sickness surveys. Of these four, only the last is considered likely to yield data which would enable statisticians to make satisfactory studies of the chronic rheumatic diseases. To be really useful, such surveys would require considerable medical and technical organization, and the home-visiting would have to be done by doctors with special training and interest in the rheumatic diseases and with facilities available for making and checking diagnoses.

Uniformity in diagnostic headings would aid in making the data from such surveys comparable from country to country; and, for this purpose, the following are suggested in the report:

Rheumatic fever

Rheumatoid arthritis (and allied conditions such as ankylosing spondylitis, Still's disease, etc.) Osteo-arthritis (arthrosis)_including the spine Other forms of arthritis-infective and traumatic Gout 
Lesions of the intervertebral disks

Non-articular rheumatism ("fibrositis")-in various parts of the body

"Rheumatism" unspecified.

A summary of statistical studies which have been made in several countries during or since the Second World War is included in an appendix to the report. Though these studies are of limited value, they give some idea of the prevalence and incidence of the rheumatic diseases. For example, in a survey made of a "probability" sample of the population of the U.S.A. in 1951, the main conclusion reached was that there were about ten million persons over the age of 14 years (i.e., about one-tenth of the total population over this age) who believed they were suffering from either "arthritis" or "rheumatism". It was estimated that about six million of these ten million persons had been told by a doctor that their complaints were due to one or another of these two conditions.

A study of rheumatic diseases as causes of disablement and of long- and short-term illnesses in relation to the social security arrangements of the metropolitan Paris area revealed that, in about 10 per cent. of 50,000 disabled persons, the cause of the disablement was a rheumatic disease, 40 per cent. of these 5,000 persons being disabled by the cardiac sequelae of rheumatic fever. About 6 per cent. of 50,000 cases of long-term illness (i.e., longer than six months but less than three years) were also attributed to rheumatic diseases, excluding cardiopathies due to rheumatic fever.

In Sweden, an inquiry made in 1943 showed that during the year $2 \cdot 5$ per 1,000 of the population had sought medical care for rheumatoid arthritis; 1.7 per 1,000, for osteo-arthritis; and 4 per 1,000 , for sciatica and fibrositis. The data also revealed that the total number of sufferers from rheumatic diseases (including rheumatic fever) that had received medical care was about 90,000. During the year, approximately 2,100 hospital beds were occupied by rheumatic patients, but it was estimated that the total number of beds needed for treatment of such patients was about 5,000 , or at least seven per 10,000 of the population.

No definite conclusions about the aetiology and pathogenesis of rheumatic diseases can be drawn from the studies made thus far. However, certain generalities emerge concerning age and sex incidence: in rheumatoid arthritis, there is a higher incidence in the middle age-groups of the female sex than in the male; there are also differences in the sex incidence of osteo-arthritis, in which incidence appears to rise steadily with age; and some studies indicate that among men laborious occupations are associated with an earlier onset of some chronic diseases with rheumatic features (e.g., lesions of the intervertebral disks) than is the case among the general population.

\section{Prevention, Control, and Treatment}

Specific preventive measures against the rheumatic diseases are as yet not feasible, except possibly against rheumatic fever, in which the streptococcal infection is probably an aetiological factor which might be countered by the use of antibiotics and of sulfonamides.

Early recognition and prompt treatment are very important. Treatment methods are largely empirical but may nevertheless yield good results. The most commonly used methods are general medical measures, including the administration of special drugs such as gold; physiotherapy; and orthopaedic techniques, including splinting, manipulation, and operative procedures. The report states that the use of active steroid and other hormones, which has recently been tried, can be of value in carefully selected cases, but must be considered as still largely in the experimental stage.

It is essential that adequate treatment facilities and hospital beds be made available for patients suffering from rheumatic diseases; that general practitioners be better informed concerning the management of rheumatic cases; that the services of specialists in these diseases be at the disposal of patients and of general practitioners; and that research on the rheumatic diseases be encouraged. Attention must also be given to limitation of disability, through patient education as well as therapeutic measures; to rehabilitation of those disabled by rheumatic diseases; and to health education of the public, which is needed to correct the erroneous belief that these diseases are incurable, practically untreatable, and usually disabling. 